\title{
Serotype-specific pneumococcal disease may be influenced by mannose-binding lectin deficiency
}

\author{
X. Vallès*\#,ף, A. Roca*, , F. Lozano+, L. Morais*, B. Suárez ${ }^{+}$, F. Casals $^{\S}$, \\ I. Mandomando*,f, B. Sigaúque*,f, D. Nhalungo*, C. Esquinas ${ }^{\#, * *}$, L. Quintó", \\ P.L. Alonso ${ }^{*}$, and A. Torres ${ }^{\#, * *}$
}

ABSTRACT: Previous studies of the association between the mannose-binding lectin pathway deficiencies and invasive pneumococcal disease are inconclusive. Invasiveness of Streptococcus pneumoniae is dependent on serotype. We aimed to determine the association between invasive pneumococcal disease and MBL2 and MASP2 genetic variants, regarding serotype distribution.

A hospital-based case-control study was conducted in children admitted to hospital in rural Mozambique in June 2002-November 2003. The study included children admitted to hospital with invasive pneumococcal disease, in whom S. pneumoniae was isolated from blood and subsequently serotyped. Sequence-based typing analysis of amplicons covering the polymorphic regions of MASP2 (exon 3) and MBL2 (promoter and exon 1) was performed.

An overall high frequency of MBL2 genotypes associated with low serum levels of MBL (43\%) was found. Carriers of MBL-deficient genotypes were associated with invasive pneumococcal disease produced by low-invasive serotypes (OR 5.55, 95\% Cl 1.4-21.9; $\mathrm{p}=0.01$ ).

Our data suggest that susceptibility to pneumococcal disease among MBL-deficient patients may be influenced by serotype invasiveness. Type-specific capsular serotype of $S$. pneumoniae would need to be taken into account in further genetic association studies of invasive pneumococcal disease.

KEYWORDS: Africa, children, invasive pneumococcal disease, mannose-binding lectin, serotype, Streptococcus pneumoniae

nvasive pneumococcal disease (IPD) is a leading cause of morbidity and mortality in children worldwide and is responsible for as many as a million deaths each year, mostly in developing countries [1]. Known risk factors for developing IPD are young age, malnutrition, overcrowding and HIV-1 infection, all of which are highly prevalent in sub-Saharan African countries [2]. Furthermore, the ability of pneumococcus to cause invasive disease is highly dependent on capsular serotype [3], and the prevalence of serotypes among IPD cases varies widely according to geographical area and age [4]. Genetic risk factors for IPD focussing on congenital deficiency of mannose-binding lectin (MBL) have been reported in populations other than subSaharan Africa with no clear conclusions [5-7].

MBL is one of the recognition modules of the lectin pathway of complement activation. MBL recognises specific carbohydrate moieties found in a wide range of microorganisms [8]. MBL can then promote either direct opsonisation and phagocytosis or complement-mediated lysis through activation of MBL-associated serine proteases (MASPs) [9, 10]. Three nonsynonymous single nucleotide polymorphisms (SNP) located in exon 1 have been described: Arg52 $\rightarrow$ Cys (D variant (rs50307370), Gly54 $\rightarrow$ Asp (B variant (rs1800450)) and Gly57 $\rightarrow$ Glu (C variant (rs1800451)). The wild type variant is considered the A variant and other structural variants will be referred to as 0 in the text. All of them impair MBL oligomerisation, which is essential for association with MASPs and to reach complete functional activity [11]. Furthermore, SNP of the promoter region at positions $-550 \quad(\mathrm{G} \rightarrow \mathrm{C}, \mathrm{H} / \mathrm{L}$ (rs11003125)), -221 $(\mathrm{G} \rightarrow \mathrm{C}, \mathrm{X} / \mathrm{Y}$ (rs7096206)) and the untranslated region $+4(\mathrm{P} / \mathrm{Q}($ rs7095891)) affect MBL2 transcription, with a dominant downregulatory effect of the

\section{AFFILIATIONS}

*Centro de Investigação em Saúde de Manhiça, and

${ }^{f}$ Instituto Nacional de Saúde, Ministerio de Saúde, Maputo, Mozambique.

\#Pneumology Dept, Clinic Institute of Thorax (ICT), Hospital Clinic of Barcelona, Institut d'Investigacions August Pi i Sunyer (IDIBAPS),

- Barcelona Centre for International Health Research (CRESIB), Hospital Clínic/IDIBAPS,

${ }^{+}$Unit of Immunology, Hospital Clínic de Barcelona/IDIBAPS, Departament de Biologia Cel.lular, Immunologia i Neurociències, University of Barcelona,

${ }^{\S}$ Institut de Biologia Evolutiva (CSICUPF), CEXS-UPF-PRBB, and

${ }^{\star *}$ CIBER de Enfermedades

Respiratorias (CIBERES), Barcelona, Spain.

CORRESPONDENCE

$X$. Vallès

Pneumology Dept

Clinic Institute of Thorax (ICT)

Hospital Clinic of Barcelona

Villarroel 170

2nd floor

08036

Barcelona

Spain

E-mail: xavier valles04@

hotmail.com

Received:

Oct 292009

Accepted after revision:

Jan 272010

First published online:

Feb 112010 
$X$ allele [12]. The SNP at the promoter and exon 1 are in strong linkage disequilibrium and give rise to a limited number of prevalent haplotypes: LYPA, LYPB, LYQA, LYQC, HYPA, HYPD and LXPA, which can predict MBL serum levels [12]. Distribution of MBL haplotypes differs throughout the world, depending on ethnic groups, and LYQC has been found in high frequencies only among sub-Saharan African populations [12]. MBL deficiency has been associated with a wide range of infectious and noninfectious diseases, specifically in children [13].

MASP-2 is the most relevant serine protease associated with MBL [14]. A frequent nonsynonymous amino acid replacement involving codon 105 (Asp $\rightarrow$ Gly (rs56392418)) of the CUB-1 domain has been found among Caucasians and has been linked to severe immunodeficiency [15]. We performed a case-control study to investigate the association between MBL2 and MASP2 variants, and IPD in children, taking into account serotype distribution. The study was carried out in children $<5$ yrs of age from a rural area in Southern Mozambique with high incidence of IPD (416 out of 100,000 per child-yr at risk) [16].

\section{MATERIALS AND METHODS}

\section{Population and study location}

The study was conducted by the Centro de Investigação em Saúde da Manhiça (CISM; Maputo, Mozambique) at Manhiça District Hospital (MDH), which has 110 beds including 26 paediatric beds; the hospital is the referral health facility for Manhiça District, a rural area of Maputo province in Southern Mozambique. Manhiça District had an estimated population of 130,000 inhabitants during the study period, with homogeneous ethnicity (Xironga and Xichangana). Details of the study location have been described elsewhere [17]. Briefly, the climate of the area is subtropical with two distinct seasons: a warm, rainy season between November and April, and a cool, dry season during the rest of the year. Malaria is endemic throughout the year, peaking between December and March. The prevalence of HIV-1 infection among pregnant females during the study period was 19\% (unpublished data).

\section{Clinical monitoring and sample collection}

Since January 1997, the MDH and the CISM have jointly performed round-the-clock monitoring of all paediatric visits to the outpatient dept and all admissions to the wards [16]. On admission, a trained medical officer complete a detailed clinical questionnaire. A finger-prick blood sample for determination of malaria parasites in thin and thick blood smears were obtained from children with fever (axillary temperature $\geqslant 37.5^{\circ} \mathrm{C}$ ), and packed-cell volume (PCV) was also measured. As part of routine clinical practice, blood cultures were performed on admission in all children $<2$ yrs of age and in older children with an axillary temperature $>39^{\circ} \mathrm{C}$.

The present study was approved by the Mozambican Ethics Review Committee (Ministry of Health, Maputo, Mozambique) and the Ethics Committee of the Hospital Clínic (Barcelona, Spain). Written informed consent was obtained from the parents or tutors of eligible children.

\section{Variable definitions}

A child with IPD was defined as a patient admitted to the MDH, in whom Streptococcus pneumoniae was isolated from blood.
Increased respiratory rate (IRR) was defined according to age group as a respiratory rate: $>60$ breaths $\cdot \mathrm{min}^{-1}$ for children under 2 months; $>50$ breaths $\cdot \mathrm{min}^{-1}$ for children aged 212 months; and $>40$ breaths $\cdot \mathrm{min}^{-1}$ for children aged $1-5 \mathrm{yrs}$ [18]. Following this definition, clinical pneumonia was diagnosed when children were admitted to hospital with cough or breathing difficulty, and IRR or chest indrawing [18]. Anaemia was classified according to levels of PCV as mild (PCV 25-33\%), moderate (PCV 15-24\%) or severe (PCV $<15 \%$ ). Children were classified as severely malnourished if their weight $\mathrm{z}$-score was $\leqslant-3 \mathrm{SD}$, and moderately malnourished with a weight $\mathrm{z}$-score of $-2--3$ SD [19]. Plasmodium falciparum infection was defined when one or more asexual parasites were seen in blood after observation of $>200$ leukocytes. Clinical malaria was defined, according to age, as fever plus any $P$. falciparum parasitaemia (in infants) or $>2,500$ parasites $\cdot \mathrm{mm}^{-3}$ (in other children). The rainy season was considered to be between November and April and the dry season was considered to be the rest of the calendar year. Mortality was defined as the number of deaths from a specific condition occurring in hospital divided by the number of children with known outcome (deaths plus discharges). Children with unknown final outcome, such as those transferred or absconding from hospital were not included in mortality measurements.

Genotypes coding for MBL high (A/A, XA/A), intermediate $(\mathrm{A} / 0, \mathrm{XA} / \mathrm{XA})$, and low $(0 / 0, \mathrm{XA} / 0)$ serum levels were defined according to previous reports $[12,20-22]$. Individuals with these genotypes will be referred to as MBL-sufficient or MBL-deficient (moderate and severe, respectively).

Highly invasive serotypes of $S$. pneumoniae were considered serotypes with an estimated attack rate of $>20$, based on a previous report that included serotypes 1, 4, 5, 9V, $12 \mathrm{~F}$ and 14 [23].

\section{Study design}

We conducted a case-control study in children aged $<5$ yrs admitted to the MDH between June 2002 and November 2003. Controls were randomly selected, at a ratio of one study case for every two controls, from children admitted to hospital during the same period with no clinical signs or symptoms of pneumonia and with a negative bacterial blood culture on admission. Criteria for matching were as follows: 1) age (frequencies \pm 6 months in children $>1 \mathrm{yr}$ of age and \pm 2 months in children $<1 \mathrm{yr}$ of age) and 2) season on admission (dry season or rainy season). Cases were stratified according to serotype invasiveness of S. pneumoniae (highinvasive versus low-invasive).

\section{Laboratory methods}

\section{MBL2 and MASP2 genotyping}

DNA was extracted and purified from biological samples using a commercial kit (Qiagen DNA blood kit, Qiagen, Valencia, CA, USA). A 969-bp fragment encompassing the promoter to the end of exon 1 of the MBL2 gene was obtained by PCR amplification using the sense 5'-GGGGAATTCCTGCCAGAAAGT-3' and antisense 5'-CATATCCCCAGGCAGTTTCCTC-3' primers and the Expand ${ }^{\mathrm{TM}}$ 20kbPLUS PCR System (Roche Diagnostics GmbH, Mannheim, Germany). A $354 \mathrm{bp}$ fragment from exon 3 of the MASP2 gene was PCRamplified using the sense 5'-GCGAGTACGACTTCGTCAAGG-3' and antisense 5'-CTCGGCTGCATAGAAGGCCTC-3' 
TABLE 1 General prevalence of different variables comparing study cases and controls

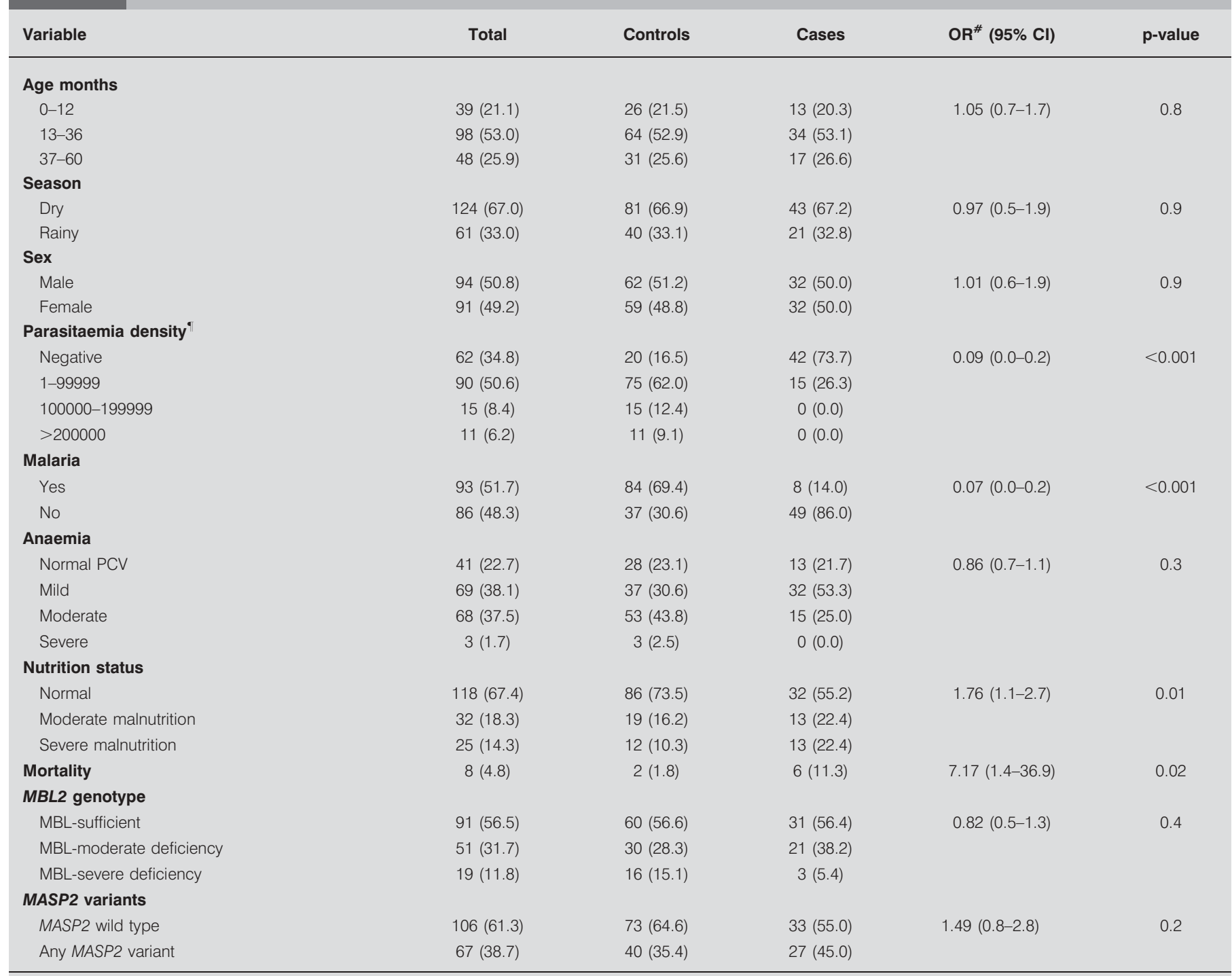

Data are presented as n (\%), unless otherwise stated. PCV: packed-cell volume; MBL: mannose-binding lectin; MASP: MBL-associated serine protease. ${ }^{\#}$ : pooled OR for one category of increased adjusted by design variables (season and age) when appropriate; " : estimated number of Plasmodium falciparum asexual parasites per mm ${ }^{3}$.

oligonucleotides and the Expand ${ }^{\mathrm{TM}}$ High Fidelity PCR System (Roche Diagnostics $\mathrm{GmbH}$ ).

The cycling conditions used for amplification of both the MBL2 and MASP2 genes were $94^{\circ} \mathrm{C}$ for $8 \mathrm{~min}, 35$ cycles of $94^{\circ} \mathrm{C}$ for $45 \mathrm{~s}, 58^{\circ} \mathrm{C}$ for $30 \mathrm{~s}, 72^{\circ} \mathrm{C}$ for $90 \mathrm{~s}$ and $72^{\circ} \mathrm{C}$ for $10 \mathrm{~min} .5 \mu \mathrm{L}$ of the resulting PCR reaction was treated with ExoSAP-IT (USB Corporation, Cleveland, $\mathrm{OH}, \mathrm{USA}$ ) and then subjected to direct sequencing using the sense and antisense gene-specific primers described above and the BigDye Terminator v1.1 Cycle Sequencing Kit (Applied Biosystems). Sequencing reactions were run on an ABI Prism 3100 Genetic Analyser (Applied Biosystems, Warrington, UK).

\section{S. pneumoniae isolation and serotyping}

Isolation of S. pneumoniae and serotyping (Quellung reaction) were performed using previously described standard procedures $[16,24]$.

\section{Statistical methods and computational analysis}

Descriptive results are expressed in proportions or mean \pm SD, as required. Chi-squared statistics were used to compare $2 \times 2$ table associations or a trend in larger tables, and the Fisher exact test was used when numbers were low $(<5$ in one category). An unpaired t-test was used when comparing two continuous variables. We performed logistic regression for the multivariate analysis and polytomous logistic regression to analyse different outcome groups (cases with highly invasive serotypes and cases with low-invasive serotypes), and to calculate OR and $95 \%$ CI. The final logistic regression model included the design variables (age and season) and variables with significant associations after the raw analysis. Allele and genotype frequencies for each variant site and HardyWeinberg equilibria were estimated. Statistically significant values were considered when $p \leqslant 0.05$. Multiple sequence alignments were performed with ClustalW software 
TABLE 2 Serotype distribution stratified by MBL2 genotype

\begin{tabular}{|c|c|c|c|c|}
\hline Serotype & MBL-deficient & MBL-sufficient & Attack rate $(95 \% \mathrm{Cl})^{\#}$ & Invasiveness OR $(95 \% \mathrm{Cl})$ \\
\hline \multicolumn{5}{|c|}{ Highly-invasive } \\
\hline 4 & 1 & 0 & $75(19-660)$ & $12.1(1.1-104)$ \\
\hline 5 & 1 & 3 & $\infty(9-\infty)$ & ND \\
\hline $9 \mathrm{~V}$ & 0 & 3 & $26(14-64)$ & $1.5(0.6-4.0)$ \\
\hline Subtotal & $9(43)$ & $20(77)$ & & \\
\hline \multicolumn{5}{|c|}{ Low-invasive } \\
\hline $6 \mathrm{~A}$ & 4 & 1 & $8(5-14)$ & $0.7(0.3-1.7)$ \\
\hline $6 B$ & 0 & 1 & $5(4-7)$ & $0.6(0.3-1.0)$ \\
\hline $10 \mathrm{~F}$ & 0 & 1 & ND & ND \\
\hline $15 \mathrm{~A}$ & 1 & 0 & $0(0-33)$ & 0 \\
\hline $23 \mathrm{~F}$ & 3 & 0 & $8(5-13)$ & $0.4(0.2-0.8)$ \\
\hline $24 \mathrm{~F}$ & 1 & 1 & ND & 0 \\
\hline 29 & 0 & 1 & ND & 0 \\
\hline Subtotal & $12(57)$ & $6(23)$ & & \\
\hline Total & 21 & 26 & & \\
\hline
\end{tabular}

Data are presented as $n$ or $n(\%)$, unless otherwise stated. MBL: mannose-binding lectin. ${ }^{\#}:$ data from SLEEMAN et al. [23]. Attack rate is defined as the number of serotypespecific IPD cases by 100,000 nasopharyngeal acquisitions. ' : data from BRUEGGEMANN et al. [3]. Invasiveness OR is defined as the relative prevalence of specific serotypes among IPD isolates and nasopharyngeal isolates.

(European Bioinformatics Institute, Cambridge, UK). Data were analysed using the STATA 10.0 software package (Stata Corp., College Station, TX, USA).

\section{RESULTS}

\section{Descriptive results}

185 children (64 cases and 121 controls) were eligible for the study. A total of 161 samples were amplified for MBL2 and 173 samples were amplified for MASP2 (recovery rate of $87 \%$ for MBL2 and 94\% for MASP2; 24 and 12 samples were not amplified, respectively, due to the poor quality of the DNA). A total of 55 cases $(86 \%)$ and 106 controls $(88 \%)$ had both MBL2 and MASP2 genotyping results. The mean $\pm \mathrm{SD}$ age was $26.4 \pm 15.8$ months, and about half of the patients were male $(51 \%)$. Malnutrition and mortality were more frequent among study cases ( 45 versus $27 \%, \mathrm{p}=0.01$, and 11 versus $2 \%, \mathrm{p}=0.02$, respectively). Malaria was predominantly diagnosed among controls $(69 \%)$ and, hence, parasitaemia was more prevalent among them. Table 1 provides a full description of prevalence of variables among cases and controls.

There were no differences between children with and without genotyping data available for all the study variables described in table 1 . Overall, 70 of the 161 children (43\%) had a genotype responsible for low MBL deficiency, and 19 out of $161(12 \%)$ had a genotype associated with severe deficiency (10 homozygous for haplotypes involving structural mutations and nine heterozygous for the LXPA haplotype combined with the LYQC haplotype). The Hardy-Weinberg equilibrium was conserved for alleles for the whole sample A/C $(p=0.48), H / L$ $(p=0.26), P / Q(p=0.82)$, and $Y / X(p=0.13)$, as well as the control group $(p=0.33, p=0.08, p=0.93$ and $p=0.28$, respectively). 67 children (38.7\%) carried a nonsynonymous MASP2 variant. Full description and prevalence of MASP2 polymorphisms and MBL2 genotypes found is reported elsewhere [25].

\section{IPD and MBL pathway variants}

Among study children, IPD was neither associated with MBL deficiency (OR 1.44, 95\% CI 0.6-3.6; $\mathrm{p}=0.4$ ) nor MASP2 variants (OR 1.59, 95\% CI 0.6-3.9; $\mathrm{p}=0.3$ ), even when examining different exposure groups (severely MBL-deficient versus normal $(\mathrm{p}=0.4)$ or moderately MBL-deficient versus normal $(\mathrm{p}=0.2))$ or analysing different MASP2 variants separately.

IPD and MBL deficiency according to serotype distribution Analysis according to serotype distribution included 47 out of $64(73 \%)$ cases with available data on serotyping and MBL2 genotyping. Serotype distribution in terms of MBL deficiency is shown in table 2 . Overall, 21 cases were MBL-deficient (18 moderately deficient and three severely deficient) and 29 were infected by a highly invasive serotype. The final logistic regression model showed that MBL deficiency was more prevalent among individuals with IPD caused by low-invasive pneumococcal serotypes than control individuals (OR 3.93, 95\% CI 1.1-14.5; $\mathrm{p}=0.04)$.

No differences in the prevalence of MBL-deficiency were detected between cases of IPD caused by highly invasive serotypes and controls (OR 0.82, 95\% CI 0.3-2.2; $\mathrm{p}=0.7$ ). 
When the dataset was stratified according to MBL deficiency (moderate and severe), results remained significant for individuals with moderate MBL deficiency infected by lowinvasive serotypes after univariate and multivariate analysis (OR 3.56, 95\% CI 1.1-.11.2, p=0.03, and OR 5.55, 95\% CI 1.4$21.9, p=0.01$, respectively). Only two cases infected by lowinvasive serotypes had severe deficiencies. Furthermore, all infants (children $<1 \mathrm{yr}$ of age) infected by low-invasive serotypes $(n=8)$ carried an MBL-deficient genotype (8 out of 8 versus 4 out of $10 ; p=0.001)$. Tables 3 and 4 shows the univariate and multivariate analysis of data stratified according to highly invasive serotypes and low-invasive serotypes, respectively. To overcome any confounding effect produced by the intermediate serotypes (serotypes with attack ratio $>20$ but large CI including the cut-off of $20(4,9 \mathrm{~V}$ and $12 \mathrm{~F}$; $\mathrm{n}=6)$, we carried out an additional analysis excluding or classifying them as low-invasive strains. We did not find any significant change in this additional analysis ( $p=0.03$ for both).
When comparing cases with highly invasive serotypes versus low-invasive serotypes, stratified by MBL genotype (sufficient, moderately deficient and severely deficient), we found an almost significant $p$-value for trend $(p=0.054)$, suggesting increased susceptibility depending on the degree of MBLdeficiency (fig. 1). No association was found when analysing MASP2 variants versus MASP2 wild type with IPD and serotype invasiveness (tables 3 and 4).

\section{DISCUSSION}

The results of our analysis raise the hypothesis that serotypespecific IPD in children may be influenced by MBL deficiency. Children with MBL deficiency may be more prone to infection by a low-invasive serotype compared with MBL-sufficient children, but not by highly invasive serotypes. The plausibility of this hypothesis is supported by the observation that opsonin activity has been shown to influence intracellular trafficking and killing of S. pneumoniae by human alveolar macrophages [26].

\begin{tabular}{|c|c|c|c|c|c|}
\hline Variable & Cases & Univariate $^{\#}$ OR $(95 \% \mathrm{Cl})$ & p-value & Adjusted OR" $(95 \% \mathrm{Cl})$ & $\mathrm{p}$-value \\
\hline \multicolumn{6}{|l|}{ Anaemia } \\
\hline Normal PCV & $6(21.7)$ & 1 & & & \\
\hline Mild & 17 (58.6) & $2.13(0.7-6.2)$ & 0.2 & & \\
\hline \multicolumn{6}{|l|}{ Nutrition status } \\
\hline Normal & $16(59.3)$ & 1 & & & \\
\hline Moderate malnutrition & 7 (25.9) & $1.95(0.7-5.4)$ & 0.2 & & \\
\hline Severe malnutrition & $4(14.8)$ & $1.83(0.6-6.6)$ & 0.4 & & \\
\hline \multicolumn{6}{|l|}{ Parasitaemia ${ }^{+, \S}$} \\
\hline No & $24(85.7)$ & 1 & & & \\
\hline Yes & $4(14.3)$ & $0.01(0.0-0.3)$ & $<0.001$ & $0.07(0.0-0.2)$ & $<0.001$ \\
\hline \multicolumn{6}{|l|}{ MBL2 genotype } \\
\hline Sufficient & $20(69.0)$ & 1 & & & \\
\hline Moderately deficient & 8 (27.6) & $0.78(0.3-2.0)$ & 0.6 & $1.19(0.4-3.5)$ & 0.7 \\
\hline Severely deficient & $1(3.5)$ & $0.19(0.0-1.5)$ & 0.1 & $0.21(0.0-2.0)$ & 0.2 \\
\hline \multicolumn{6}{|l|}{ Age months } \\
\hline $0-12$ & $3(10.3)$ & 1 & & & \\
\hline $13-36$ & $19(65.5)$ & $2.50(0.7-9.2)$ & 0.2 & $2.31(0.5-10.3)$ & 0.3 \\
\hline $37-60$ & $7(24.1)$ & $2.01(0.5-8.8)$ & 0.4 & $1.17(0.2-6.7)$ & 0.9 \\
\hline \multicolumn{6}{|l|}{ Season } \\
\hline
\end{tabular}

Data are presented as $n(\%)$, unless otherwise stated. PCV: packed-cell volume; MBL: mannose-binding lectin; MASP: MBL-associated serine protease. * : adjusted by the design variables (when appropriate) of age and season; " : the final logistic model included design variables (age and season), malaria and MASP2 variants; ${ }^{+}$: all subjects with parasitaemia $\geqslant 100,000$ were controls and analysis could not be carried out; ${ }^{\text {s. }}$ : parasitaemia was excluded from final logistic model analysis due to colinearity with clinical malaria; ${ }^{f}$ : includes subjects with all data available (MBL2 and MASP2 genotyping). 


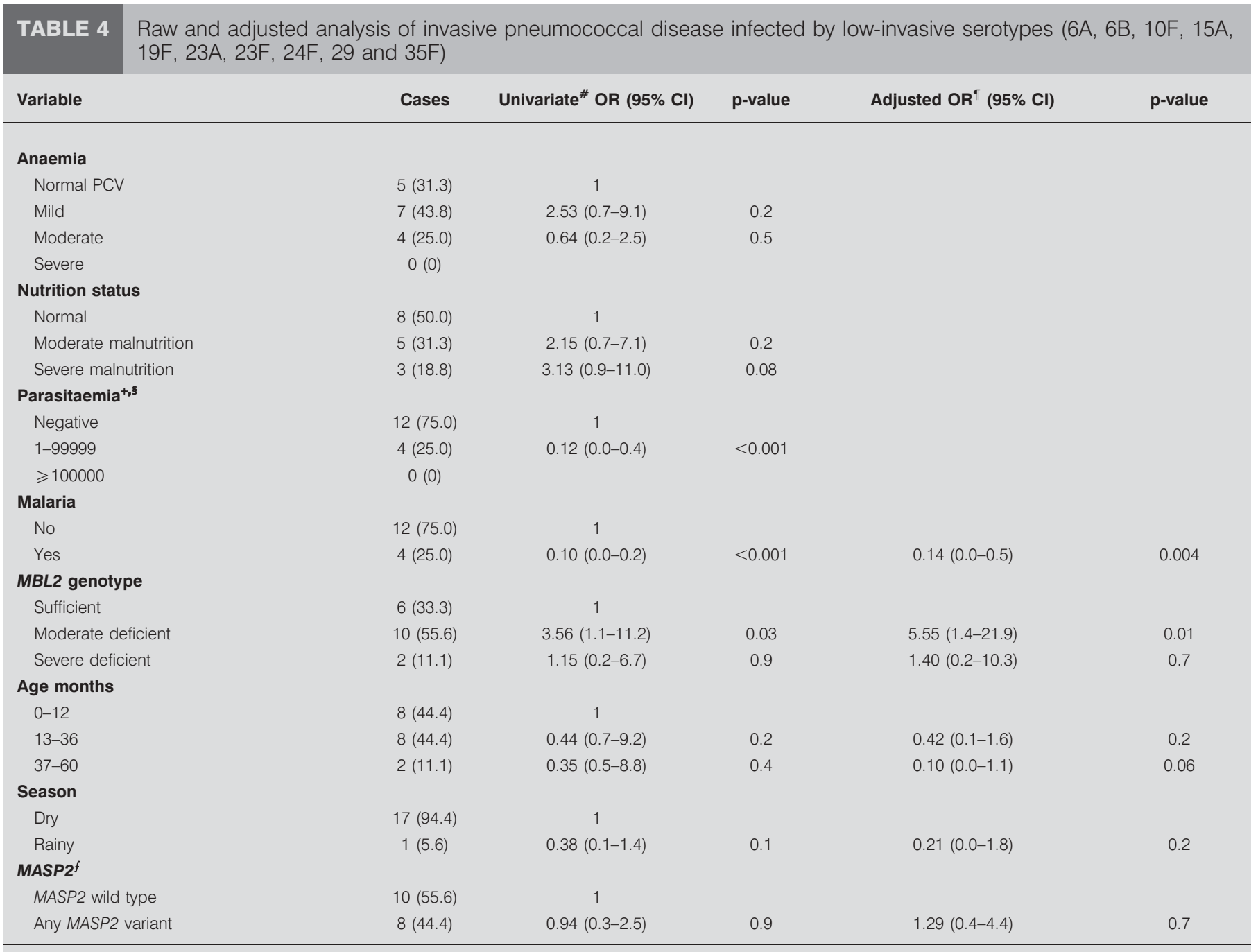

Data are presented as $n(\%)$, unless otherwise stated. PCV: packed-cell volume; MBL: mannose-binding lectin; MASP: MBL-associated serine protease. ${ }^{\#}$ : adjusted by the design variables (when appropriate) of age and season; ${ }^{\bullet}$ : the final logistic model included design variables (age and season), malaria and MASP2 variants; ${ }^{+}:$all

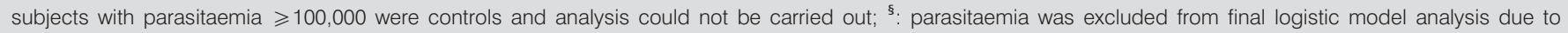
colinearity with clinical malaria; ${ }^{f}$ : includes subjects with all data available (MBL2 and MASP2 genotyping).

The ability of highly invasive serotypes to escape opsonophagocytosis may be the main strategy used. In contrast, lowinvasive serotypes would need predisposing host factors (such as MBL deficiency) to cause disease, compared with the truly primary pathogens (i.e. highly invasive serotypes), which would be less influenced by any opsonophagocytosis deficiency. It has been shown that highly invasive serotypes act independently of other comorbidities in contrast to the low-invasive or opportunistic serotypes [27].

If the association between MBL deficiency and IPD depends on serotype invasiveness, the chances of observing an association (without taking into account serotype distribution in the final analysis) in a well-conducted study would largely depend on the prevalence of different serotypes. Associations could be diluted and significances would be found in large studies. This would explain the discordant findings in previously published studies where serotype distribution was not taken into account [5-7].
Furthermore, it has been reported that manifestations of MBL deficiency would be more prominent in young children, whose immune system is still immature [28]. This notion is supported by the over-representation of subjects with MBL-deficiency in infants infected by low-invasive serotypes.

Alternatively, HIV infection might explain the observed association between MBL deficiency and low-invasive serotypes. HIV has been established as a strong risk factor for pneumonia and IPD $[2,29]$. Recent data from the same hospital show a $26 \%$ prevalence of HIV infection among children under 2 years of age admitted to hospital between March 2005-2006 with severe pneumonia [30]. Taking into account that $S$. pneumoniae is the leading cause of severe pneumonia in children, we can assume that the prevalence of HIV infection among cases included in our study would be remarkable. MBL-deficient genotypes have been shown to influence vertical transmission of HIV infection and AIDS progression, 


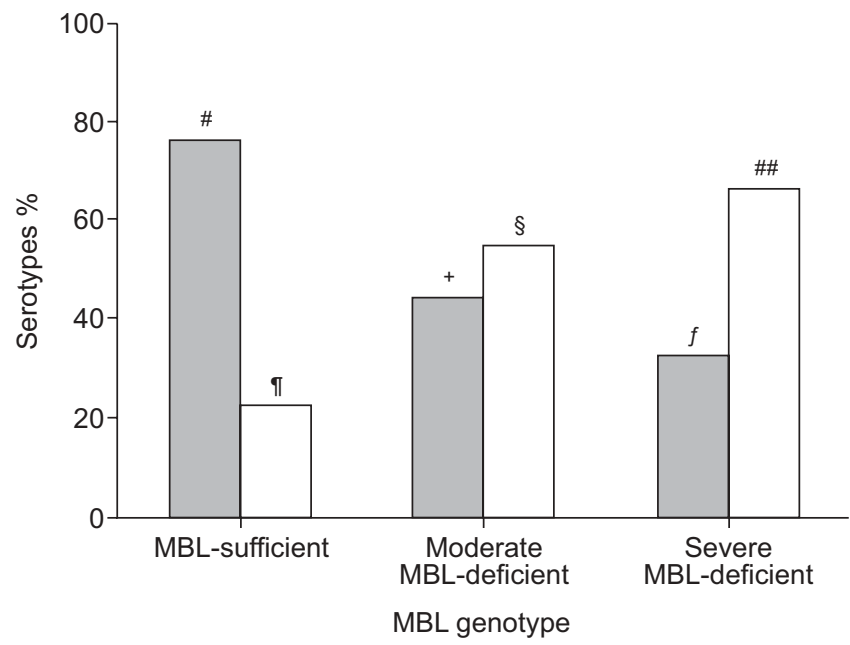

FIGURE 1. MBL genotype distribution according to serotype invasiveness among cases. Capsular serotype-specific distribution as follows. ${ }^{*}: 1(n=9), 5$ $(n=3), 9 \vee(n=3), 14(n=5) ; ": 6 A(n=1), 6 B(n=1), 10 F(n=1), 22 A(n=1), 24 F$ $(n=1), 19(n=1) ;{ }^{+}: 1(n=4), 4(n=1), 5(n=1), 12 F(n=1), 14(n=1) ;{ }^{5}: 6 A(n=3)$, 15A $(n=1), 19 F(n=2), 23 A(n=1), 23 F(n=2), 24 F(n=1) ;{ }^{f}: 12 F(n=1) ;{ }^{\# \#: 6 A}$ $(\mathrm{n}=1)$ and $23 \mathrm{~F}(\mathrm{n}=1)$. $\square$ high-invasive; $\square$ : low-invasive

as well as AIDS progression among HIV-infected children [31]. HIV-infected individuals may have higher susceptibility to less invasive serotypes than other children. Consequently, the association between MBL deficiency and low-invasive serotypes may be confounded by HIV infection. However, HIV contribution should decrease with age, being insignificant for older cases (5-15 yrs of age), as mortality for HIV-infected children is high when treatment for this age group is scarce. This might explain the over-representation of MBL deficiency among infants (children $<1 \mathrm{yr}$ of age) infected by a lowinvasive serotype. Further studies are needed to explore this hypothesis, as HIV data were not available from children included this study.

In conclusion, our results suggest the hypothesis that serotype invasiveness in IPD in children may be influenced by MBL deficiency. This observation is in accordance with current knowledge of physiopathology and epidemiology of IPD, may explain the inconclusive results of previous studies [5-7], and can inspire future studies focused on IPD and genetic risk factors. Further studies taking into account the limitations mentioned in our design (such as the small numbers, the lack of HIV data, and the hospital-based source of controls) are necessary to confirm these findings and to determine whether these results can be extrapolated to other geographical areas.

\section{SUPPORT STATEMENT}

This study was funded by 2009 SGR 911, Ciber de Enfermedades Respiratorias (Ciberes CB06/06/0028), the Ciberes is an initiative of the ISCIII. The work by F. Lozano and B. Suárez is funded by the Spanish Research Network on Infectious Diseases (REIPI; RD06/0008/1013). The preliminary results of this study were presented at the 2006 conference of the American Thoracic Society (May 2006). The work by A. Roca was supported by grants from the Spanish Ministry of Science and Innovation (Ramón y Cajal: RYC-2008-02777).

\section{STATEMENT OF INTEREST}

None declared.

\section{ACKNOWLEDGEMENTS}

The authors wish to thank laboratory and clinical staff from the CISM and $\mathrm{MDH}$ (Maputo, Mozambique) for their role in collecting and processing clinical samples. We thank A. Andrés, A. Ramírez-Soriano and J. Bertranpetit (Unitat de Biologia Evolutiva, Universitat Pompeu Fabra, Barcelona, Spain), V. Moreno and K. Louie (Institut Català d'Oncologia, l'Hospitalet de Llobregat, Spain) and J.J. Aponte (Barcelona Centre for International Health Research, Barcelona, Spain) for their advice and contributions.

\section{REFERENCES}

1 Scott JA. The preventable burden of pneumococcal disease in the developing world. Vaccine 2007; 25: 2398-2405.

2 Schuchat A, Dowell SF. Pneumonia in children in the developing world: new challenges, new solutions. Semin Pediatr Infect Dis 2004; 15: 181-189.

3 Brueggemann AB, Griffiths DT, Meats E, et al. Clonal relationships between invasive and carriage Streptococcus pneumoniae and serotype- and clone-specific differences in invasive disease potential. J Infect Dis 2003; 187: 1424-1432.

4 Hausdorff WP, Feikin DR, Klugman KP. Epidemiological differences among pneumococcal serotypes. Lancet Infect Dis 2005; 5: 83-93.

5 Roy S, Knox K, Segal S, et al. MBL genotype and risk of invasive pneumococcal disease: a case-control study. Lancet 2002; 359: 1569-1573.

6 Kronborg G, Weis N, Madsen HO, et al. Variant mannose-binding lectin alleles are not associated with susceptibility to or outcome of invasive pneumococcal infection in randomly included patients. J Infect Dis 2002; 185: 1517-1520.

7 Moens L, Van Hoeyveld E, Peetermans WE, et al. Mannosebinding lectin genotype and invasive pneumococcal infection. Hum Immunol 2006; 67: 605-611.

8 Jack DL, Klein NJ, Turner MW. Mannose-binding lectin: targeting the microbial world for complement attack and opsonophagocytosis. Immunol Rev 2001; 180: 86-99.

9 Matsushita M, Fujita T. Activation of the classical complement pathway by mannose-binding protein in association with a novel C1s-like serine protease. J Exp Med 1992; 176: 1497-1502.

10 Ji $\mathrm{YH}$, Fujita T, Hatsuse $\mathrm{H}$, et al. Activation of the $\mathrm{C} 4$ and $\mathrm{C} 2$ components of complement by a proteinase in serum bactericidal factor, RA reactive factor. J Immunol 1993; 150: 571-578.

11 Larsen F, Madsen HO, Sim RB, et al. Disease-associated mutations in human mannose-binding lectin compromise oligomerization and activity of the final protein. J Biol Chem 2004; 279: 21302-21311.

12 Garred P, Larsen F, Seyfarth J, et al. Mannose-binding lectin and its genetic variants. Genes Immun 2006; 7: 85-94.

13 Thiel S, Frederiksen PD, Jensenius JC. Clinical manifestations of mannan-binding lectin deficiency. Mol Immunol 2006; 43: 86-96.

14 Sorensen R, Thiel S, Jensenius JC. Mannan-binding-lectin-associated serine proteases, characteristics and disease associations. Springer Semin Immunopathol 2005; 27: 299-319.

15 Stengaard-Pedersen K, Thiel S, Gadjeva $\mathrm{M}$, et al. Inherited deficiency of mannan-binding lectin-associated serine protease 2 . N Engl J Med 2003; 349: 554-560.

16 Roca A, Sigaúque B, Quintó L, et al. Epidemiological and clinical characteristics of invasive pneumococcal disease in children less than 5 years of age in rural Mozambique. Trop Med Int Health 2006; 11: $1422-1431$.

17 Nhacolo AQ, Nhalungo DA, Sacoor CN, et al. Levels and trends of demographic indices in southern rural Mozambique: evidence from demographic surveillance in Manhiça district. BMC Public Health 2006; 11: 1741-1748.

18 WHO young infants study group. Clinical prediction of serious bacterial infections in young infants in developing countries. Pediatr Infect Dis J 1999; 18: s23-s31. 
19 Shann F. Nutritional indices: z, centile, or percent? Lancet 1993; 27: 84-85.

20 Steffensen R, Thiel S, Varming K, et al. Detection of structural gene mutations and promoter polymorphisms in the mannan-binding lectin (MBL) gene by polymerase chain reaction with sequencespecific primers. J Immunol Methods 2000; 241: 33-42.

21 Minchinton RM, Dean MM, Clark TR, et al. Analysis of the relationship between mannose-binding lectin (MBL) genotype, MBL levels and function in an Australian blood donor population. Scand J Immunol 2002; 56: 630-641.

22 Kilpatrick DC. Mannan-binding lectin: clinical significance and applications. Biochim Biophys Acta 2002; 1572: 401-413.

23 Sleeman KL, Griffiths D, Shackley F, et al. Capsular serotype-specific attack rates and duration of carriage of Streptococcus pneumoniae in a population of children. J Infect Dis 2006; 194: 682-688.

24 Vallès X, Flannery B, Roca A, et al. Serotype distribution and antibiotic susceptibility of invasive and nasopharyngeal isolates of Streptococcus pneumoniae among children in rural Mozambique. Trop Med Int Health 2006; 11: 358-366.

25 Vallès X, Sarrias MR, Casals F, et al. Genetic and structural analysis of MBL2 and MASP2 polymorphisms in south-eastern African children. Tissue Antigens 2009; 74: 298-307.
26 Gordon SB, Irving GR, Lawson RA, et al. Intracellular trafficking and killing of Streptococcus pneumoniae by human alveolar macrophages are influenced by opsonins. Infect Immun 2000; 68: 2286-2293.

27 Sjöström K, Spindler C, Ortqvist A, et al. Clonal and capsular types decide whether penumococci will act as a primary or opportunistic pathogen. Clin Infect Dis 2006; 42: 451-459.

28 Koch A, Melbye M, Sorensen $\mathrm{P}$, et al. Acute respiratory tract infections and mannose-binding lectin insufficiency during early childhood. JAMA 2001; 285: 1316-1321.

29 Madhi SA, Petersen K, Madhi A, et al. increased disease burden and antibiotic resistance of bacteria causing severe communityacquired lower respiratory tract infections in human immunodeficiency virus type 1-infected children. Clin Infect Dis 2000; 31: 170-176.

30 Sigaúque B, Roca A, Bassat $\mathrm{Q}$, et al. Severe pneumonia in Mozambican young children: clinical and radiological characteristics and risk factors. J Trop Pediatr 2009; 55: 379-387.

31 Mangano A, Rocco C, Marino SM, et al. Detrimental effects of mannose-binding lectin (MBL2) promoter genotype XA/XA on HIV-1 vertical transmission and AIDS progression. J Infect Dis 2008; 198: 694-700. 